\title{
Method of thermally stimulated acoustic emission to assess changes in the deformed state of rocks under quasi-static loading
}

\author{
R. Oshkin \\ National University of Science and Technology MISIS, Mining Institute, Moscow, Russia
}

\begin{abstract}
This paper presents the results of an experimental study of the acoustic-emission response of limestone samples exposed to incrementally increasing quasi-static mechanical loads and a series of thermal shocks. The subject of the study also included the design and composition (configuration) of the laboratory facility for the research, as indicated above. The patterns of change in thermally stimulated acoustic emission (TAE) as a function of the deformed state of limestone under development are theoretically substantiated. An acoustic-emission criterion enabling a qualitative assessment of the residual strength of rocks was proposed and substantiated. The methodological approaches to the processing with the presentation of the author's interpretation of the physical meaning of the experimentally obtained initial measurement data are defined. Finally, the fundamental possibility of using the obtained results in the field conditions for monitoring the evolution of the deformed state of rocks is shown.
\end{abstract}

\section{Introduction}

At present, several deposits of mineral resources which are relatively easy to find during exploration, tend to deplete. Both first and production mining become deeper and more intense, which leads to a higher risk of slips, falls, rock bumps, and other dynamic rock pressure manifestations [1]. Mitigation of these risks requires justification and use of efficient preventive measures to ensure control of rock conditions. A precondition to solving the problem is the availability of reliable and time-sensitive information on the stress-strain behavior (SSB) of the geological environment. Geo-control methods [2, 3, 4], existing and available at present, although they have some advantages, are also not without disadvantages, among which labor-intensive measurements and ambiguity of interpretation can be singled out.

Practically, these disadvantages result in poorer informational efficiency of geocontrol and higher risks of overlooking the signs of hazardous geodynamic conditions. In this connection, a critical task is to improve existing methods and create new ones, which could be used both individually and in conjunction with conventional geological, geo-mechanical, and geophysical methods to control SSB. Recent research work shows the prospects of solving this and other geocontrol-related problems based on the analysis of informative parameters of acoustic emission, thermally stimulated within the geomaterials to be investigated $[5,6,7,8]$. 
Given the above-mentioned, the purpose of the present paper is to experimentally determine and theoretically justify patterns of changes to parameters of thermally stimulated acoustic emission of rocks, subject to the dynamics of their deformed state. It is expected that in the long term, these patterns will, enable the implementation of an appropriate method for SSB control directly within the rock masses, exposing the borehole environments to thermal stresses and analyzing the corresponding acoustic-emission response.

\section{Instrumentation and methodological support of the experiments}

For the experiments was used limestone originating from the Sary-Tash deposit, samples of which were in the form of parallelepipeds with square cross-section and polished ends. The length of the base sides of the samples ranged from 22.0 to $25.6 \mathrm{~mm}$, and their height varied between 48.8 and $51.3 \mathrm{~mm}$. In all cases, the ratio of the base side and height was as close to 1:2 as possible. All the samples were made from one piece of relatively homogeneous rock and inspected for internal defects using a UD2-16 instrument (ultrasound pulsecounting method). The homogeneity of the samples was confirmed by a visual inspection of their outer surfaces, for which a portable microscope was used ("DigiMicro Mobile"). The inspection revealed no significant surface defects.

For the determination of strength properties of the limestone subject to research, four samples were tested in accordance with GOST 21153.2 for fracture strength when exposed to uniaxial compression $\left(\sigma_{\text {comp }}\right)$. The following results of $\sigma_{\text {comp }}$ were obtained: $27.5 \mathrm{MPa}$; 30.6 MPa; 20.5 MPa; 28.7 MPa. The sample with 20.5 MPa was identified using the ultrasound-based method, as suspected for having a masked main crack. Other samples exhibited no abnormal crack-like defects. With this consideration in mind, a value of $28.9 \mathrm{MPa}$ was taken as an average to establish the increments of quasi-static mechanical loads and further interpretation of the results obtained.

Experiments were carried out using a laboratory facility, the design and composition (configuration) of which are presented in Figure 1. 


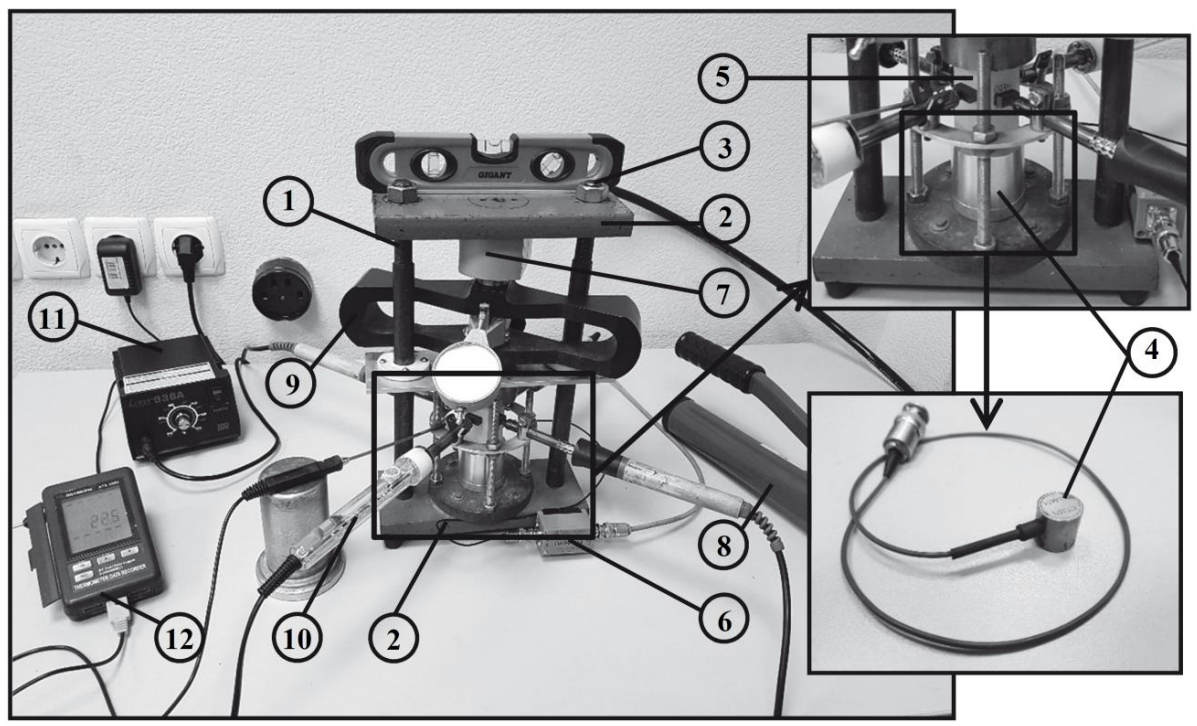

Fig. 1. The exterior appearance of the laboratory facility used to apply thermomechanical loads on rock samples and record parameters of the acoustic emission occurring.

The facility includes a metal framework, assembled with the use of guide bars (1), tops and bottoms of which are connected with plates (2). The guide bars are fixed with locking nuts (3). In the center of the lower metal plate (2), there is an acoustic emission converter, model GT-200, emplaced inside a metal casing (4) of a cylindrical shape, on top of which a rock sample (5) is placed. The acoustic emission-converter GT-200 is connected to an amplifier ALP 01 (6), from which a cable runs to a multi-channel modular system (A-line 32D) that gathers and processes acoustic-emission data (in Figure 1, the system is not shown conventionally). The placed sample takes a uniaxial load from a DN10P11 (7) hydraulic jack, powered by a manually-operated hydraulic pump KVT PMR 7003 (8). To control the loading procedure, an analogous dynamometer, type DS-5 (9) placed beneath the jack, is used. Thermal loads were created using heating elements (10), adjacent to the central parts of each free face of the sample tested. Heat transmission parameters were adjusted using a Lukey 936A thermal station equipped with a temperature regulator (11), or, depending on the design of heating elements, with voltage supplied through a laboratory transformer. Control of temperature conditions of samples surface and heating elements operation during the experiments was carried out using a temperature measuring and recording device ATE-9380 (12), equipped with a group of thermocouples ATA-210.

In the course of the experiments, the samples were exposed to thermomechanical loads, which were mechanical in nature and incrementally increasing to values of $4,6,8,10,12$, $14 \mathrm{kN}$, subjected to fast heating to $180^{\circ} \mathrm{C}$ with further convection cooling at each increment of load. The samples were thermally exposed while stabilizing the acoustic emission (AE) burst activity, following the mechanical loads as indicated above, which allowed to separate the useful signal from the interference. To simulate the pressure drop in the rocks before each increment of mechanical loading, the previous maximum load value had to be halved. The selected loading mode is shown in Figure 2. 


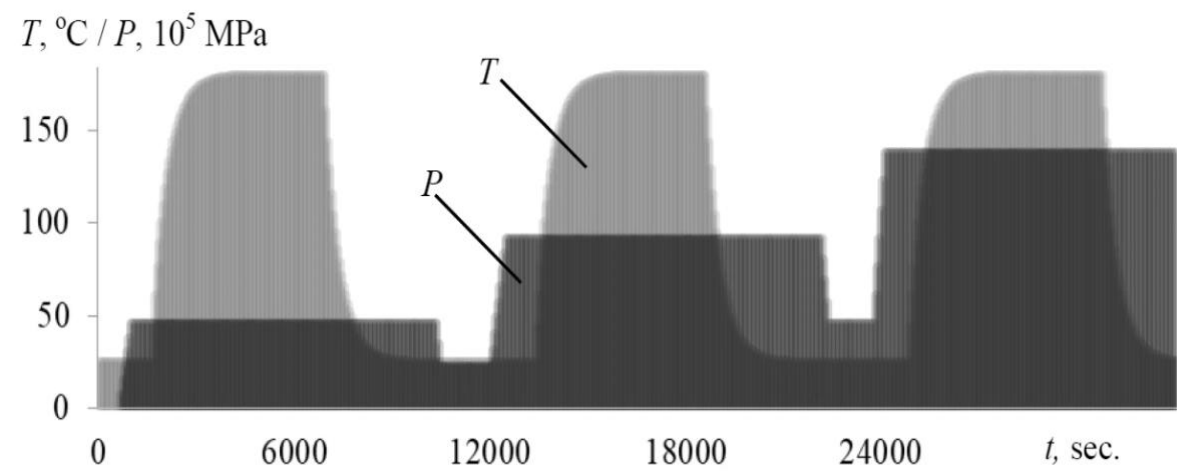

Fig. 2. Typical fragment of exposure, where: $T$ is the sample surface temperature at a point located $10 \mathrm{~mm}$ away from the heating element; $P$ is the value of mechanical load; $\mathrm{t}$ is the experiment starting time.

\section{Processing and interpretation of the experimental results}

Figure 3 shows a representative example of initial measurement data to be acquired during the experiments.

There are two underlying mechanisms of AE signal generation. The first one is related to the destruction of the structural bonds of geomaterials in the broadest sense: crack formation and growth, fracture of rock pore walls, deformation of mineral grains, resulting from uneven expansion of the surrounding rocks when exposed to a temperature gradient, etc. The second mechanism is the transition of structural bonds to the deformed state and their further relaxation to their initial state after removal of the temperature field. In this case, the following illustrative example can be provided: one crack is adjacent to the wall of another one. The propagation of the first crack stops until the stress concentrator has accumulated enough energy, to coalesce the cracks at the contact point. In other words, almost no fracture occurs within that area until cracks are merged, and the AE pulses are not generated due the resistance of rock to increased stresses ("stretched string effect").

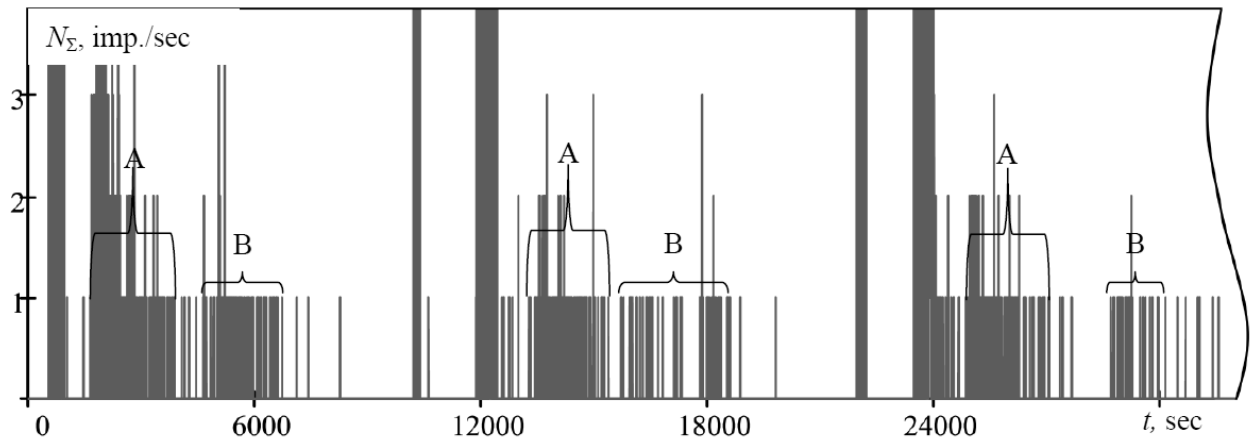

Fig. 3. A representative type of distribution of AE $\dot{N}_{\Sigma}$ signals activity, where: A is the time domain of AE signals generation, stimulated by a field of variable temperatures (thermal gradient); B is the time domain of AE signals generation, stimulated by a field of stationary temperatures with samples heated to a maximum temperature. 
The "A" domain (Figure 3) is dominated by the fracture mechanism. Due to the destruction of structural bonds, weakened by the preliminary mechanical impact, the thermally generated stresses are redistributed and partially relaxed. As the resources of these bonds deplete, and the temperature field stabilizes, i.e., as the thermal stresses become stationary (domain "B"), the nature of TAE is mainly affected by the mechanism of structural bond transition of to a stress state, with their integrity preserved.

Thus, comparing the AE response parameters in "A" and "B" domains allows us to quantify the ratio of structural bonds, preliminarily destroyed by mechanical loading and bonds with their integrity preserved and sufficient for relaxation into the initial state after the partial removal of loads.

The following informative parameters were used: AE activity $N_{\Sigma}$ and pulse duration $D_{\text {imp }}$, which were averaged by time marks within "A" and "B" domains. The physical meaning of $N_{\Sigma}$ as a parameter, indicating the number of events recorded per unit of time, is a measure of structural bonds that emit TAE signals. The term "TAE signal" shall be understood to mean an elastic wave, resulting from the reaction of rocks to thermomechanical stresses within them, which is described by various AE parameters to be recorded by the Aline 32D. In its turn, the $D_{\text {imp }}$ parameter characterizes the average duration of TAE signals. Under other similar conditions, the structural bonds can emit a proportionally longer signal. Therefore, the $D_{\mathrm{imp}}$ value of allows to separate those TAE signals in the signal stream that relate to strong bonds. Apparently, the latter determine the strength properties of rocks to a far greater degree, and information on their destruction is of the vital importance for the evaluation of residual strength of geomaterials.

Against this background, the following acoustic-emission criterion $R$ was proposed to quantify the residual strength of rocks by the example of the limestone samples test results:

$$
R=\frac{N_{\sum}^{\mathrm{A}} \times D_{\mathrm{imp}}^{\mathrm{A}}}{N_{\sum}^{\mathrm{B}} \times D_{\mathrm{imp}}^{\mathrm{B}}}
$$

where $N_{\Sigma}^{\mathrm{A}}, D_{\text {imp }}{ }^{\mathrm{A}}$ and $N_{\Sigma}^{\mathrm{B}}, D_{\text {imp }}{ }^{\mathrm{B}}$ are values of $N_{\Sigma}, D_{\text {imp }}$, averaged by the time of "A" and "B" domains, correspondingly.

Physically, the $R$ criterion is meant to reflect the degree of rock resistance to external factors. In this particular case, the only variable factor was the value of the mechanical load. Thermal impacts in all experiments were the same, and the identity of the samples in the structure and properties was validated with the use of visual, optical, and ultrasound methods. Therefore, the $R$ criterion can be reasonably considered as a criterion for evaluation of the residual strength of limestone exposed to increasing quasi-static mechanical loads.

Figure 4 presents a cloud of the obtained $R$-values as a function of mechanical loads $P$ used for obtaining these values. 


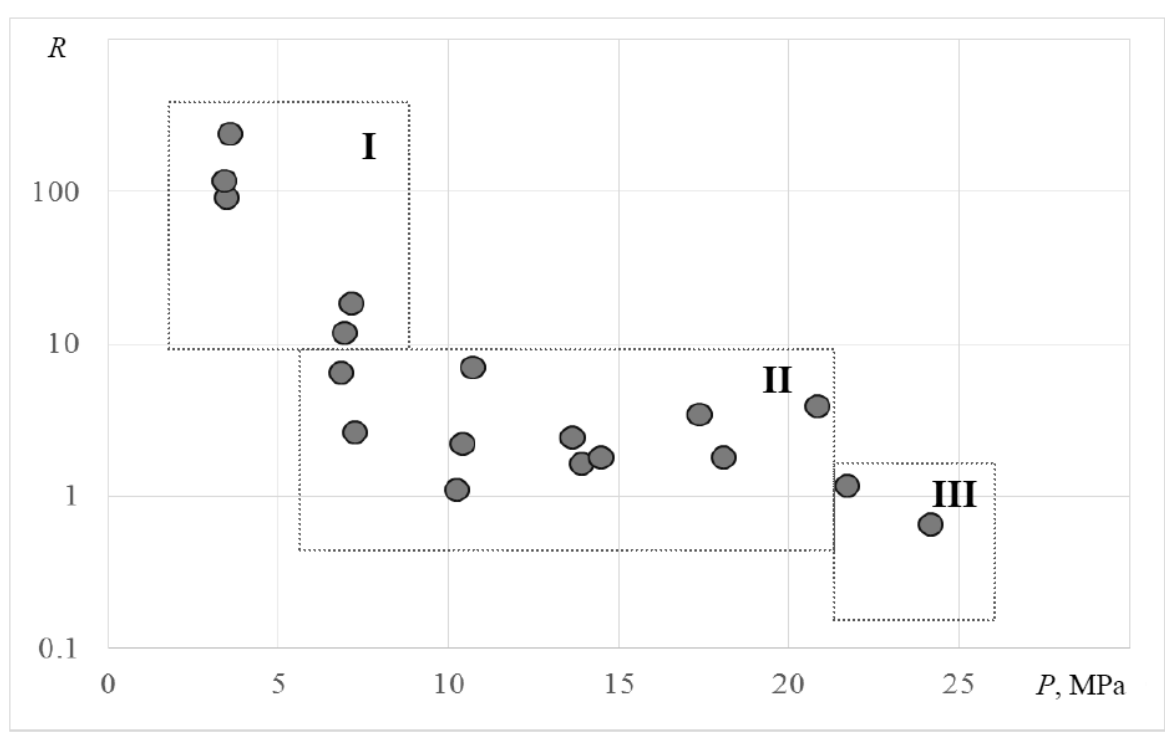

Fig. 4. Relationship between the acoustic-emission criterion $R$ and values of mechanical loads $P$, with which the values of $R$ were obtained.

Stage 1 (Figure 4) consists of the fast destruction of weak structural bonds, initially contained in the samples, as well as collapses of pores and cavities. The foregoing results from a particularity of the acoustic-emission response at this stage, which is characterized by a higher intensity of the acoustic emission in the "A" domain with relatively high average values of $D_{\text {imp. }}$. This means that a great number of weak bonds are subject to destruction, which is rather fast but creates no conditions for the generation of long-duration TAE pulses.

As the resources of weak structural bonds deplete, another stage begins, which is the stabilization of the rock structure (Stage II). The deformed state develops according to a law, which is similar to the linear one. But this is an accumulation of stresses that prevails over destruction processes, before the merging of cracks into a single crack network and division of the sample into pieces. The latter process takes place at Stage III. The small number of points here results from the technical complexity of adjusting such a load onto the sample, so that branching and merging of cracks would begin but lead to no fracture of the sample until the end of acoustic-emission measurements (their duration is about 1.5 ...2.0 hours).

\section{Conclusion}

The experimentally proven relationship between thermally stimulated acoustic emission parameters in the limestone samples and the evolution of their deformed state under the influence of increasing quasi-static mechanical loads is presented and theoretically substantiated. The physical preconditions underlying this relationship are interpreted and allow to substantiate a new acoustic-emission criterion for quantative assessment of the residual strength of rocks. The fundamental possibility of using this criterion for detecting signs of rapid fracture of the tested object is shown.

The results obtained may be used both for developing a new acoustic-emission related method for monitoring the strength properties of rocks and for finding solutions to forecast problems related with the assessment and evaluation of hazardous geodynamic conditions 
during performance of primary mining (expensed development), underground mining of mineral resources and other mining operations.

\section{References}

1. V.N. Karelin, A.V. Kravchenko, L.L. Yu., B.P. Kazakov, A.V. Zaitsev, Features of the formation of microclimatic conditions in mining samples of a deep mining camp. Gornyi Zhurnal 6: 65-68. (2013).

2. J. Jaeger, N.G. Cook, R. Zimmerman, Fundamentals of Rock Mechanics. (Wiley/Blackwell, 2007).

3. V.L. Shkuratnik, P.V. Nikolenko, Methods for determining the stress-strain state of a rock massif: scientific and educational course. (Moscow: MSMU, 2012).

4. V.N. Tyupin, T.I. Rubashkina, Blasting Methods of Stress State Determination in Rock Mass. Journal of Mining Science, 54: 569-574. (2018).

5. E.A. Novikov, V.L. Shkuratnik, R.O. Oshkin, M.G. Zaitsev, Effect of the stress-strain state of sandy-clay soils on their thermally stimulated acoustic emission. Soil Mechanics and Foundation Engineering 54 (2): 81 - 86. (2017).

6. V.L. Shkuratnik, E.A. Novikov, R.O. Oshkin, Experimental analysis of thermally stimulated acoustic emission in various-genotype rock specimens under uniaxial compression. Journal of Mining Science, 50: 249 - 255. (2014).

7. V.L. Shkuratnik, E.A. Novikov, A.S. Voznesenskii, V.A. Vinnikov, Thermostimulated acoustic emission in geomaterials (monograph). (Moscow : Gornaya Kniga, 2015).

8. V.L. Shkuratnik, E.A. Novikov, Thermally stimulated acoustic emission of rocks as a promising tool of geocontrol, .Gornyi Zhurnal, 6: 21-26. (2017). 